\title{
Validez de constructo y confiabilidad de la APGAR familiar en pacientes odontológicos adultos de Cartagena, Colombia
}

\section{Construct validity and reliability of the family APGAR in adult dental patients from Cartagena, Colombia}

\author{
Shyrley Díaz-Cárdenas ${ }^{1}$; Lesbia Tirado-Amador²; Miguel Simancas-Pallares ${ }^{1}$
}

Forma de citar: Díaz-Cárdenas S, Tirado Amador L, Simancas-Pallaeres M. Validez de constructo y confiabilidad de la APGAR familiar en pacientes odontológicos adultos de Cartagena, Colombia. Rev Univ Ind Santander Salud. 2017; 49(4): 541-548. doi: http://dx.doi.org/10.18273/revsal.v49n4-2017003 (c)

\section{Resumen}

Objetivo: Determinar la validez de constructo y confiabilidad del APGAR familiar en pacientes odontológicos adultos de Cartagena, Colombia. Métodos: Estudio de validación de escalas sin criterio de referencia en 227 pacientes entre 22 y 94 años atendidos en una Clínica Odontológica Universitaria del Norte (Colombia). Los datos del APGAR se extrajeron a partir de la historia clínica de salud familiar. La consistencia interna se estimó con la fórmula de Kuder-Richardson y la validez de constructo, empleando análisis factorial exploratorio (AFE) y confirmatorio (AFC). Los siguientes índices de ajuste se obtuvieron a partir del AFC: $\chi 2$, p-valor para $\chi 2$, grados de libertad (gl), raíz cuadrada del error medio de aproximación, índice comparativo de ajuste e índice de Tucker-Lewis. El análisis de los datos se hizo a través de Stata v.13.2 para Windows (StataCorp, TX, USA) y Mplus v.7.31 para

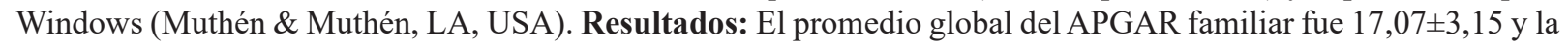
prevalencia de disfunción familiar fue 44,4 \% (IC 95\%: 38 - 51 \%). La consistencia interna fue 0,90. El AFE reportó un solo factor que explicó el 64,1 \% de la varianza total. El AFC mostró como índices de ajuste $\chi 2=702,960 ; \mathrm{gl}=10$, p-valor=0.001; RCEMA=0.155 (IC90\%: 0.107-0.209); ICA=0.961; ITL=0.922. Conclusiones: En la muestra, APGAR familiar resultó ser un instrumento de tamizaje con alta utilidad clínica, excelente consistencia interna pero cuestionable validez de constructo. Deben realizarse investigaciones adicionales a fin de obtener escalas con adecuadas propiedades psicométricas para la medición de constructos en salud.

Palabras clave: Familia; Psicometría; Epidemiología; Puntaje de Apgar.

\begin{abstract}
Objective: To determine construct validity and reliability of the family APGAR scale in dental adult patients from Cartagena, Colombia. Methods: Scale validation study without a reference criterion in 227 patients between 22 and 94 years old whom consulted a college-based dental clinic. Family APGAR data was obtained from the family health charts. Internal consistency was calculated with the Kuder-Richardson formula and construct validity, by

1. Universidad de Cartagena, Bolivar. Colombia.

2. Universidad del Sinú, Cartagena. Colombia.

Correspondencia: Miguel Simancas-Pallares. Calle 30 \# 48-152. Universidad de Cartagena. Campus Ciencias de la Salud, Facultad de Odontología. Departamento de Investigaciones - Oficina 301. Correo electrónico: msimancasp@unicartagena.edu.co. Teléfono: +57 5 6698172. Ext: 110.
\end{abstract}


means of exploratory (EFA) and confirmatory factor analysis (CFA). Fit indexes were obtained from CFA: $\chi 2$; p-value for $\chi 2$; degrees of freedom (df); root mean square error of approximation (RMSEA); comparative fit index (CFI) and Tucker-Lewis index (TLI). Data analysis was performed using Stata v.13.2 for Windows (StataCorp, TX, US) and Mplus v.7.31 for Windows (Muthén \& Muthén, LA, US). Results: Family APGAR overall score was $17,07 \pm 3,15$ and prevalence of family dysfunction was 44,4\% (95\% CI: $38-51 \%)$. Internal consistency was 0,90 . EFA showed a single factor that accounted for $64,1 \%$ of the total variance. CFA showed as fit indexes: $\chi 2=702,960$; $\mathrm{df}=10, \chi 2$ p-value $=0.001$; RMSEA $=0.155$ (90\% CI: 0.107-0.209); CFI=0.961; TLI $=0.922$. Conclusions: Family APGAR in Cartagena dental adult patients seems to be a highly valuable screening instrument with excellent internal consistency but questionable construct validity. Additional studies must be conducted in order to obtain scales with adequate psychometric properties for construct measurement.

Keywords: Family; Psychometrics; Epidemiology; Apgar Score.

\section{Introducción}

La familia se entiende como la unidad bio-psicosocial de la sociedad y una de sus funciones vitales es contribuir a la salud de todos sus miembros por medio de la transmisión efectiva de creencias y valores, así como del soporte brindado. En este sentido, todo el proceso que acontece desde que se reconoce la enfermedad hasta que se cura o desaparece está influenciado por las decisiones que se adoptan en el seno del grupo familiar $^{1,2}$.

La salud bucal como parte de la salud integral del individuo también se encuentra influenciada por estas decisiones y soporte que ofrece la familia. Existe evidencia que indica que la presencia de disfunción familiar sugiere mayor riesgo de sufrir caries dental en dentición primaria (RP: 1,47. IC $95 \%$ : $1,11-1,95)^{3}$. Estudios locales reportan que la falta de recursos como espacio, tiempo y dinero están relacionados con la presencia de caries de infancia temprana $(\mathrm{P} \text {-valor }=0,04)^{4}$. Estos aspectos como la falta de recursos económicos son conclusivos al asociarse con la funcionalidad familiar. Así, para lograr un buen estado de salud bucal son necesarios recursos económicos para la compra de elementos de higiene y para aprender hábitos adecuados de higiene bucal se precisa también de espacios y tiempos al interior de las familia ${ }^{4}$. De aquí la importancia valorar la funcionalidad familiar en pacientes que asisten a la consulta odontológica puesto que la medición de estos aspectos resulta de alto valor para el diagnóstico, tratamiento y pronóstico de la enfermedad ${ }^{5}$.

Uno de los instrumentos más empleados a nivel mundial para la medición de la percepción de la funcionalidad familiar es la escala APGAR familiar. Propuesto originalmente por Smilkstein en 1978 y luego validado en 1982, se ha convertido en una herramienta rápida de tamizaje de los cinco componentes de la función familiar: Adaptación, Participación, Ganancia o Crecimiento, Afecto y Recursos (APGAR) ${ }^{6,7}$. En esta escala, cada componente se expresa con una pregunta evaluada a través de un formato de respuesta tipo Likert. La adaptación se refiere al uso de recursos intra y extrafamiliares para solución de problemas cuando el equilibrio de la familia se afecta por una crisis. La participación se entiende como la toma de decisiones compartidas y la alimentación de responsabilidades mutua. Por su parte, la ganancia o crecimiento es la maduración física y emocional y la autorrealización de los miembros de la familia a través de soporte recíproco. El afecto describe una relación de cariño o cuidado que existe al interior de la familia. Finalmente, la resolución es el compromiso de invertir tiempo u otros recursos con los miembros de la familia; también incluye una decisión de compartir salud y espacio físico ${ }^{6}$.

En su reporte original de validación en inglés, la APGAR mostró una adecuada consistencia interna (Alfa de Cronbach=0,86) ${ }^{7}$. En 1994 se propuso en Colombia mostrando buena validez de apariencia pero sin reportes de consistencia interna $y / 0$ validez $^{2}$. Subsecuentemente, se adaptó al Español (en España) mostrando adecuada consistencia interna $(\alpha=0,84)$ y a través de análisis factorial exploratorio (AFE), evidenció la presencia de un solo constructo: función familiar ${ }^{1}$. Otras versiones disponibles en español se reportan en Perú $^{8}$ y portugués en Brasil ${ }^{9}$, mostrando adecuada consistencia interna $(\alpha=0,729$ para español en Perú y 0,80 para portugués en Brasil) y ratificando la presencia de un solo factor. En Colombia hay investigaciones que reportan el uso de la escala pero pocos han evaluado su desempeño psicométrico ${ }^{10,11}$. En 2006 Campo-Arias, et al. en población del oriente del país, determinaron su consistencia interna $(\alpha=0,79)$ y a través de AFE, la APGAR mostró un solo factor que dió cuenta del $55 \%$ de la varianza sugiriendo entonces el aislamiento de un 
constructo unidimensional consistente con su propuesta original y otras validadas previamente. No obstante, no se contó con indicadores estadísticos sobre su validez de constructo (VC).

Los abordajes estadísticos para la valoración de VC son escasos y en sentido práctico, difíciles de aplicar ${ }^{12}$; uno de ellos es el análisis factorial confirmatorio (AFC). Perteneciente a la familia de modelado de ecuaciones estructurales, se muestra robusto frente a otras estrategias y es de creciente necesidad y por tanto uso en psicometría ${ }^{13-15}$. Actualmente no se dispone de resultados de investigaciones en Colombia que den cuenta de la VC de la APGAR empleando técnicas como el AFC. Teniendo en cuenta la recomendación de la necesidad de contar con instrumentos de adecuadas propiedades psicométricas ante diversas aplicaciones en contextos cultural y clínicamente distintos ${ }^{12,16}$, como por ejemplo pacientes con afecciones bucales, debido a que se ha analizado previamente el desempeño psicométrico en pacientes con síndrome de intestino irritable mostrando inadecuada validez discriminante. Poniendo de manifiesto toda la anterior evidencia, el objetivo del presente estudio fue determinar la validez de constructo y consistencia interna de la APGAR familiar en pacientes odontológicos adultos de Cartagena Colombia.

\section{Materiales y métodos}

Se diseñó un estudio de validación de escalas sin criterio de referencia. Los datos de la investigación se extrajeron de forma retrospectiva de las historias clínicas odontológicas de una institución universitaria del norte de Colombia, específicamente el componente de salud familiar; la cual hace parte de la historia clínica dental integral en esa institución. Este componente de Salud Familiar incluye la APGAR Familiar para lo cual se tuvieron en cuenta aquellas escalas completamente diligenciadas, con previa verificación y aprobación de su diligenciamiento por parte del Docente-Tutor, Especialista del área.

La APGAR se entregó a pacientes mayores de 18 años, precisando que el paciente tuviese un tiempo mínimo de permanencia en el hogar de cinco años y fue autodiligenciado por el mismo paciente sin ayuda de otras personas. Sólo se seleccionaron aquellas historias clínicas que correspondieran a pacientes que no presentaran deficiencias cognitivas o motoras, enfermedades previas como hipertensión, diabetes, depresión o cualquier enfermedad que pueda modificar los resultados de la escala y que en el momento que acudió a la consulta presentase solo afecciones bucales, con el ánimo de proporcionar la mayor homogeneidad muestral. Todos los pacientes firmaron un consentimiento informado que autorizaba el tratamiento de datos de forma anónima para investigaciones y perteneciente a un protocolo previamente aprobado por el comité de ética en investigación de la institución.

\section{Emplazamiento y características de la población}

Se incluyeron 227 pacientes entre 22 y 94 años atendidos en la clínica odontológica de una institución universitaria al norte de Colombia con edad promedio de $62,08 \pm 10,5$ años y en su gran mayoría mujeres $(63,1$ $\%$ ). Respecto de las condiciones sociodemográficas el $60,44 \%$ era desempleado, $34,6 \%$ pertenecían a estrato 3 y en su gran mayoría casadoas $(65,78 \%)$.

\section{Instrumento}

La APGAR es un instrumento que consta de cinco preguntas como: 1) "Me siento satisfecho con la ayuda que recibo de mi familia cuando tengo algún problema o necesidad?" y 2) "Me siento satisfecho con la forma en que mi familia habla de las cosas y comparte los problemas conmigo?"que indagan por el estado de la funcionalidad familiar en los últimos seis meses. En su versión original en Inglés, la escala ofrecía tres opciones de respuesta a estas preguntas en un formato Likert (casi siempre $=2$, algunas veces $=1$ y casi nunca $=0$ ). Sin embargo, investigaciones posteriores concluyeron que un formato de respuesta con mayor número de categorías (nunca $=0$, casi nunca $=1$, algunas veces $=2$, algunas veces $=3$ y casi siempre $=4$ ) mejoraba su desempeño psicométrico $(\alpha=0,86)$ y por tanto la capacidad de medición del constructo ${ }^{7}$. En este sentido, el puntaje mínimo de la escala puede ser 0 y el máximo 20. Puntajes superiores a 17 sugieren buena función familiar. La versión propuesta en 1994 en español para Colombia ${ }^{2}$ fue la empleada en este estudio y que ha sido objeto de investigaciones sobre su desempeño psicométrico $^{17}$.

\section{Análisis de Datos}

Inicialmente se realizó estadística descriptiva. Para las variables cualitativas se calcularon proporciones e intervalos de confianza al $95 \%$ y para variables continuas media y desviación estándar o mediana y rango intercuartílico dependiendo de la simetría de la distribución (test de Shapiro Wilks). La evaluación de las propiedades psicométricas incluyó: consistencia interna y validez de constructo. 
La consistencia interna se estimó con la fórmula 20 de Kuder-Richardson y se evaluó de acuerdo a los criterios propuestos por Kline como sigue: aceptable $(0,60$ $-0,70)$, buena $(0,70-0,90)$ y excelente $(>0,90)^{18}$. Para la evaluación de la validez de constructo inicialmente se determinó la factorizabilidad de la matriz con la prueba de esfericidad de Barlett y la prueba de adecuación de la muestra de Kaiser-Meyer-Olkin (KMO). La prueba de Barlett es un estadístico de alta sensibilidad al tamaño de la muestra y es apropiada si muestra $\chi^{2}$ alto y $\mathrm{p}$-valor $<0,05$. KMO es una prueba más conservadora, que se entiende aceptable si muestra un valor $>0,70$. Posteriormente, se realizó AFE con la técnica de máxima verosimilitud para conocer la dimensionalidad del constructo (número de factores, dimensiones o dominios). El número de factores a extraer se determinó a través de los valores propios (criterio de Kaiser: autovalores $\geq 1$ ) y un gráfico de sedimentación de Catell (parallel analysis). No obstante, el gráfico de sedimentación muestra ser un mejor parámetro respecto del criterio de Kaiser el cual sobre-estima el número de factores a retener ${ }^{19}$. Para definir la conformación de factores, se tomaron en cuenta las cargas factoriales $\geq 0,40^{14}$. Se empleó rotación oblicua (promax) puesto que se hipotetizó que los posibles factores estarían correlacionados entre si $^{20}$.

Para proceder con el AFC, inicialmente se verificó la normalidad multivariada empleando el Test de Mardia.
Aunque no se encontró normalidad multivariada, el AFC es robusto a este tipo de supuestos cuando se tienen tamaños de muestra amplios $(\geq 200)$ y no existe normalidad multivariada ${ }^{21,22}$. El AFC se realizó para confirmar la estructura de factores previamente estimada en el AFE y así la validez de constructo con el método de estimación Diagonally Weighted Least Squares (WLSMV). Para la evaluación del ajuste del modelo se determinaron los siguientes estadísticos: $\chi^{2}$ y p-valor para $\chi^{2}$, grados de libertad $(g l)$, raíz cuadrada del error medio de aproximación -RCEMA e intervalo de confianza al $90 \%$, índice comparativo de ajuste - ICA e índice de Tucker-Lewis - ITL. La evaluación del ajuste del modelo se realizó empleando los criterios propuestos por $\mathrm{Hu} \&$ Bentler así: p-valor para $\chi^{2}>0,05, \operatorname{RCEMA} \leq 0,06$, ICA e ITL $>0,95^{23}$. El análisis descriptivo, el AFE y la consistencia interna se calcularon empleando Stata v.13.2 para Windows (StataCorp., TX., USA) y el AFC se realizó empleando Mplus v.7.31 para Windows (Muthén \& Muthén., Los Angeles, CA., USA).

\section{Resultados}

La media global para la APGAR fue $17,07 \pm 3,15$ (Rango 0 - 20). La estadística descriptiva para cada ítem se muestra en la Tabla 1. La prevalencia de alguna forma de disfunción familiar fue $44,4 \%$ (IC 95\%: 38 - 51).

Tabla 1. Estadística descriptiva para los reactivos componentes de la escala.

\begin{tabular}{|c|c|c|c|}
\hline Ítem & Media & Desv. Est. & CIPT \\
\hline $\begin{array}{l}\text { ¿Me siento satisfecho con la ayuda que recibo de mi familia cuando tengo algún problema o } \\
\text { necesidad? }\end{array}$ & 3,28 & 0,83 & 0,746 \\
\hline $\begin{array}{l}\text { ¿Me siento satisfecho con la forma en que mi familia habla de las cosas y comparte los } \\
\text { problemas conmigo? }\end{array}$ & 3,37 & 0,72 & 0,821 \\
\hline $\begin{array}{l}\text { ¿Me siento satisfecho con la forma como mi familia acepta y apoya mis deseos de emprender } \\
\text { nuevas actividades? }\end{array}$ & 3,42 & 0,70 & 0,742 \\
\hline $\begin{array}{l}\text { ¿Me siento satisfecho con la forma como mi familia expresa afecto y responde a mis emociones } \\
\text { como rabia, tristeza o amor? }\end{array}$ & 3,47 & 0,72 & 0,758 \\
\hline $\begin{array}{l}\text { ¿Me siento satisfecho con la manera como compartimos en mi familia: el tiempo para estar } \\
\text { juntos, los espacios en la casa, el dinero? }\end{array}$ & 3,52 & 0,72 & 0,711 \\
\hline
\end{tabular}

Desv. Est: desviación estándar. CIPT: correlación ítem-puntuación total.

\section{Consistencia Interna}

La fórmula 20 de Kuder-Richardson estimada a través del coeficiente alfa de Cronbach fue 0,90 .

\section{Validez de Constructo}

La prueba de esfericidad de Barlett en el AFE fue $\chi^{2}=692,02, g l=10$ y $p$-valor $=0.001$ y la prueba de adecuación muestral de Kaiser-Meyer-Olkin 0,86. La estructura factorial sugirió un solo factor que agrupó los cinco ítems y mostró un autovalor de 3,20 que explicó el $64,12 \%$ de la varianza total. Esto se evidenció en el gráfico de sedimentación de Catell de la Figura 1 y en la Tabla 2 se plasmó la solución factorial obtenida.

El AFC arrojó como resultado los siguientes índices de ajuste: $\quad \chi^{2}=702,960 ; \quad g l=10, \quad$ p-valor $=0.001$; 
RCEMA=0.155 (IC90\%: 0.107-0.209); ICA=0.961; ITL $=0.922$ y no se detectaron índices de modificación. La Figura 1 sugirió retener un solo factor como respuesta a la estructura factorial de la escala; allí se muestra solo uno de los valores propios por encima del punto de inflexión de la línea del número de factores desde donde esta se vuelve estable.

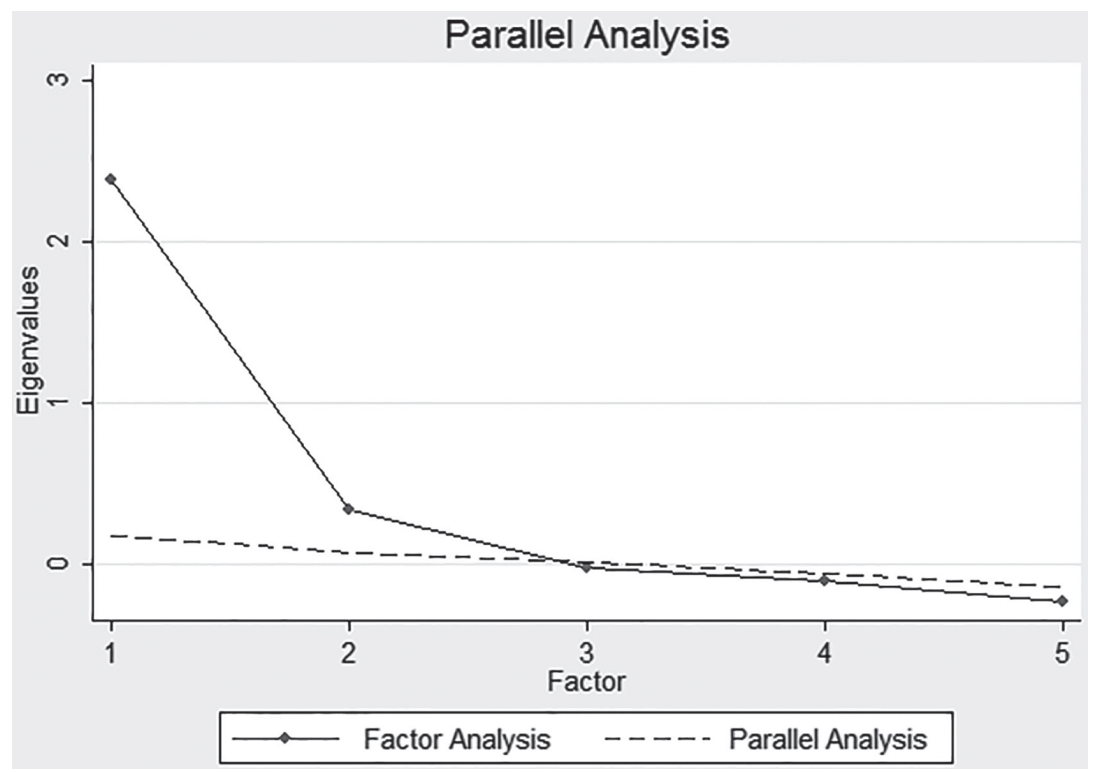

Figura 1. Gráfico de Sedimentación (de Catell) que sugiere retener un solo factor.

Tabla 2. Solución factorial obtenida para la escala APGAR familiar. Se muestran cargas factoriales y comunalidades.

\begin{tabular}{lcc}
\hline \multicolumn{1}{c}{ Ítem } & Factor & Comunalidad \\
\hline $\begin{array}{l}\text { ¿Me siento satisfecho con la ayuda que recibo de mi familia cuando tengo algún problema } \\
\text { o necesidad? }\end{array}$ & 0,804 & 0,683 \\
$\begin{array}{l}\text { ¿Me siento satisfecho con la forma en que mi familia habla de las cosas y comparte los } \\
\text { problemas conmigo? }\end{array}$ & 0,870 & 0,772 \\
$\begin{array}{l}\text { ¿Me siento satisfecho con la forma como mi familia acepta y apoya mis deseos de emprender } \\
\text { nuevas actividades? }\end{array}$ & 0,777 \\
$\begin{array}{l}\text { ¿Me siento satisfecho con la forma como mi familia expresa afecto y responde a mis } \\
\text { emociones como rabia, tristeza o amor? }\end{array}$ & 0,796 \\
$\begin{array}{l}\text { ¿Me siento satisfecho con la manera como compartimos en mi familia: el tiempo para estar } \\
\text { juntos, los espacios en la casa, el dinero? }\end{array}$ & 0,751 \\
\hline
\end{tabular}

\section{Discusión}

Desde la investigación en salud familiar resulta relevante aumentar esfuerzos por el estudio de las propiedades psicométricas de las escalas empleadas en diversos estudios, específicamente aquellas que midan la percepción del funcionamiento familiar; toda vez que la familia se constituye en el núcleo indispensable para la salud del individuo al comportarse como factor protector pero también pudiéndose comportar como factor de riesgo para la salud de sus integrantes. Así, dado que el objetivo del presente estudio fue determinar la consistencia interna y validez de constructo de la APGAR familiar, de acuerdo con los resultados, muestra ser una escala con buena consistencia interna al ser utilizada en pacientes odontológicos, lo cual refleja la buena correlación entre los cinco ítems que la componen. Estos hallazgos a pesar de reportarse en población adulta sugieren un comportamiento similar de la escala en estudiantes de secundaria como lo reportaron Forero, et al. en Bucaramanga, Colombia, aunque con valores de alpha de Cronbach inferiores $(0,79)$ a los reportados aquí en este estudio $(0,90)^{17}$. Asimismo, los valores de consistencia interna de la escala del presente estudio corresponden a valores superiores a los encontrados en la validación en inglés $(0,86)^{7}$, español en Perú $(0,72)^{8,24}$, portugués en Brasil $(0,73-0,78)^{9}$ y Portugal $(0,86)^{25}$; pero no todos los estudios reportan 
resultados de análisis factorial tanto exploratorio (AFE) como confirmatorio (AFC) para dar cuenta de sus propiedades psicométricas, específicamente validez de constructo $^{12}$. Es importante advertir que los anteriores estudios que se reportan para discutir los resultados de la presente investigación, no corresponden a estudios realizados en pacientes Odontológicos dado que no hay evidencia científica sobre el desempeño psicométrico de la APGAR en este tipo de pacientes. Sin embargo, coinciden con lo reportado en la literatura en relación al buen comportamiento de la escala.

Los resultados del AFE sugieren que la APGAR familiar sigue siendo una escala que representa un constructo unidimensional, lo cual es consistente con el reporte original de la escala ${ }^{7}$, estudios a nivel local e incluso internacional ${ }^{1,8,17}$. No obstante, el AFC desarrollado mostró pobre ajuste de los índices de evaluación del modelo lo cual sugiere una cuestionable validez de constructo de la escala. Las investigaciones previas indican que sólo existe un estudio en Perú que ha reportado previamente validez de constructo empleando técnicas de AFC y quienes evidenciaron buen ajuste del modelo obtenido $\left(\chi^{2}\right.$ : 6.783; $g l$ : 5; RCEMA: 0,04 ; TLI: 0,967 y CFI: 0,991$)^{8}$, no siendo consistente con lo aquí reportado. La discrepancia entre estos dos estudios radica en el método de estimación empleado en el AFC. Los autores de dicho estudio justifican emplear máxima verosimilitud (maximum likelihood - ML) dada la ausencia de normalidad multivariante. No obstante este método asume que los indicadores observados son continuos y que siguen una distribución multivariante normal; lo cual no siendo apropiado para variables ordinales (como los puntajes obtenidos de la APGAR familiar), puede arrojar índices de ajuste erróneos. Así, el método empleado en el presente estudio -WLSMVmuestra ser menos sesgado y más exacto respecto de ML al estimar cargas factoriales e índices de ajuste derivados de los modelos de $\mathrm{AFC}^{22}$.

Toda la anterior evidencia sugiere que la APGAR sigue comportándose como un instrumento de tamizaje que ofrece luces frente a alguna problemática por la que esté atravesando la familia, a pesar de que su uso en estudios epidemiológicos sobre pacientes como los aquí estudiados pueda representar un posible sesgo de medición dada la cuestionable validez de constructo encontrada. La forma como se diligencia esta escala es importante para evitar la introducción de estos sesgos del investigador o del trabajador de campo; sólo cuando la persona no está en capacidad de leer, lo puede hacer el entrevistador. Si el individuo puede leer, existe una contraindicación absoluta, debido a que influye el tono de la voz, la comunicación no verbal difícil de controlar en el investigador, y las presiones involuntarias que él mismo puede ejercer ${ }^{2}$. Esto no representó un sesgo en el presente estudio dada la forma auto-administrada en la cual la APGAR familiar se suministró a los participantes.

Muchas escalas como el índice de función familiar propuesto por Pless y Satterwhite con 15 ítems ${ }^{26}$, la escala de evaluación de adaptabilidad y cohesión familiar -FACES III- de 20 ítems $^{27}$, y el inventario de funcionamiento familiar de McMaster con 60 ítems $^{8}$, muestran iguales o mejores propiedades psicométricas frente a la APGAR Familiar. No obstante, la APGAR sigue siendo una de las escalas más usadas para evaluar percepción de funcionamiento familiar por tratarse de un instrumento corto, de fácil entendimiento y diligenciamiento independiente del nivel cultural y la edad del paciente. Es importante destacar que su uso radica principalmente en el área de la medicina y la enfermería y muy poco en odontología. Estudios en esta última área confirman también su utilidad teniendo en cuenta que la familia puede significar una importante covariable de estudio para la salud bucal del individuo ${ }^{29,30}$. De aquí que la condición bucal podría modificar algunos comportamientos de los procesos dinámicos relacionados con la salud familiar o viceversa, a diferencia de otros aspectos o enfermedades que podrían necesitar más de otros aspectos como el afecto y la participación familiar, como se reporta en algunos estudios donde familias con pacientes con depresión y cáncer precisan de más soporte social y comunicación $^{31,32}$, mientras que cuando se relaciona la disfuncionalidad familiar con afecciones bucales se notan frecuencias más altas negativas hacia los aspectos relacionados con la presencia de recursos como tiempo, espacio y dinero ${ }^{4}$. Por ello se tuvo especial cuidado en la selección de escalas de aquellos pacientes con solo afecciones bucales y no otras enfermedades que pueden comprometer el comportamiento sicométrico de la escala. Es importante anotar que el buen comportamiento de la escala para esta población específica de adultos con afecciones odontológicas permite recomendar su uso, sobre todo cuando se quiere conocer que aspectos específicos de la función familiar pueden comprometer la salud bucal del individuo.

No haber desarrollado un proceso de adaptación transcultural del instrumento por parte del equipo de investigadores representa una limitación en este estudio. Esto supone haber adoptado una traducción de un contexto culturalmente distinto al cual fue aplicado y que, parcialmente, también puede explicar 
la falta de ajuste en el AFC. No obstante, presenta algunas fortalezas como su tamaño de muestra pues para estudios de validez factorial es recomendable que por cada ítem existan al menos 10 sujetos $^{20}$, lo cual significaría 50 sujetos en total en el presente estudio. Además, el robusto procedimiento estadístico empleado garantiza la precisión de sus resultados ${ }^{21}$.

Toda la anterior evidencia pone de manifiesto que se debe promover ampliamente el uso de la escala en pacientes odontológicos resaltando así la activa participación familiar en la consecución de un buen estado de salud oral. Asimismo, es importante continuar la investigación sobre las propiedades psicométricas de la escala a fin de generar constructos válidos que ofrezcan mejores aportes sobre el estudio de disfuncionalidad familiar. Es importante tener en cuenta que la funcionalidad familiar responde a un proceso dinámico que requiere reajustes constantes, lo que probablemente amerite incorporar nuevos aspectos en estos instrumentos de acuerdo a la complejidad de su medición por los diversos indicadores involucrados en el funcionamiento familiar, de manera que no solo mida la presencia o no de disfuncionalidad utilizando un concepto lineal (causa-efecto) sino más bien a la luz del concepto sistémico de lo que la familia representa. El funcionamiento familiar debe verse entonces de manera circular, es decir, lo que es causa puede pasar a ser efecto o consecuencia y viceversa. El enfoque sistémico permite entonces sustituir el análisis causa-efecto por el análisis de las pautas y reglas de interacción familiar recíproca, que permitirá a su vez llegar al centro de los conflictos familiares y por tanto a las causas de la disfunción familiar. Esto representaría la ausencia de un criterio único para la medición del constructo y que deben tenerse en cuenta para la mejora de las propiedades psicométricas del instrumento ${ }^{33}$.

\section{Conclusiones}

APGAR familiar en pacientes odontológicos del norte de Colombia muestra ser un instrumento de tamizaje de alta utilidad clínica, excelente consistencia interna pero cuestionable validez de constructo. Esto ratifica que los esfuerzos en investigación sobre esta escala y específicamente para esta población deben continuar a fin que pueda seguir siendo empleado en diversos estudios epidemiológicos futuros donde se relacionen aspectos familiares y la salud bucal de los individuos.

\section{Conflicto de intereses}

Ninguno declarado por los autores.

\section{Referencias}

1. Bellón Saameño JA, Delgado Sánchez A, Luna del Castillo JD, Lardelli Claret P. Validez y fiabiliad del cuestionario de función familiar Apgar familiar. Atención Primaria. 1996; 18(6): 289-296.

2. Arias L, Herrera JA. El APGAR familiar en el cuidado primario de la salud. Colombia Médica. 1994; 25(1): 26-28.

3. Sanders AE, Slade GD. Apgar score and dental caries risk in the primary dentition of five years old. Aust Dent J. 2010; 55(3): 260-267.

4. Díaz-Cárdenas S, Arrieta-Vergara K, RamosMartínez K. Funcionalidad familiar y caries dental en niños de una institución educativa de Cartagena de Indias. Rev Col Inv Odontol. 2015; 16(6): 41-49.

5. Díaz-Cárdenas S, Tirado-Amador L, Madera-Anaya M. Odontología con enfoque en salud familiar. Rev Cub Sal Pública. 2014; 40(3): 397-405.

6. Smilkstein G. The family APGAR: a proposal for a family function test and its use by physicians. J Fam Pract. 1978; 6(6): 1231-1239.

7. Smilkstein G, Ashworth C, Montano D. Validity and reliability of the family APGAR as a test of family function. J Fam Pract. 1982; 15(2): 303-311.

8. Castilla Cabello H, Caycho Rodríguez T, Ventura León J, Palomino-Barboza M, De La Cruz Valles M. Análisis factorial confirmatorio de la escala de percepción de funcionamiento familiar de Smilkstein en adolescentes peruanos. Sal Sociedad. 2015; 6(2): 140-153.

9. da Silva MJ, Fonseca Victor J, do Nascimento Mota FR, Silva Soares E, Belém Leite BM, Teixeira Oliveira E. Analysis of psychometric properties of family APGAR with elderly in northeast Brazil. Es Anna Nery. 2014; 2014(18): 3.

10. Díaz-Cárdenas S, Arrieta-Vergara K, GonzálezMartínez F. Violencia intrafamiliar y factores de riesgo en mujeres afrodescendientes de la ciudad de Cartagena. Rev Clín Medic Familia. 2015; 8(1): 19-30.

11. Díaz-Cárdenas S, Arrieta-Vergara K, GonzálezMartínez F. Estrés académico y funcionalidad familiar en estudiantes de odontología. Sal Uninorte. 2014; 30(2): 121-132.

12. Sanchez R, Echeverry J. Validating scales used for measuring factors in medicine. Rev Sal Publica. 2004; 6(3): 302-318.

13. Harrington D. Confirmatory Factor Analysis. 1 ed. New York: Oxford University Press; 2009. 121.

14. Simancas-Pallares MA, Díaz-Cárdenas S, BarbosaGómez P, Buendía-Vergara MB, Arévalo-Tovar LL. Propiedades psicométricas del Índice de Bienestar 
General-5 de la Organización Mundial de la Salud en pacientes parcialmente edéntulos. Rev Fac Med 2016; 64(4): 701-705.

15. Simancas-Pallares M, Arrieta KM, Arévalo LL. Validez de constructo y consistencia interna de tres estructuras factoriales y dos sistemas de puntuación del Cuestionario General de Salud-12. Biomédica. 2017; 37(3).

16. Beaton DE, Bombardier C, Guillemin F, Ferraz MB. Guidelines for the process of cross-cultural adaptation of self-report measures. Spine (Phila Pa 1976). 2000; 25(24): 3186-3191.

17. Forero Ariza LM, Avendaño Durán MC, Duarte Cubillos ZJ, Campo-Arias A. Consistencia interna y análisis de factores de la escala APGAR para evaluar el funcionamiento familiar en estudiantes de básica secundaria. Rev Col Psiquiatría. 2006; 35(1): 23-29.

18. Kline P. Handbook of psychological testing: Routledge; 2013.

19. Tom AB, Bosker RJ. Multilevel Analysis: An Introduction to Basic and Advanced Multilevel Modeling. 2nd ed. Stawski RS, editor. Los Angeles, CA.: Sage; 2012. 354.

20. Costello AB, Osborne, J. Best practices in exploratory factor analysis: four recommendations for getting the most from your analysis. Practical Assessment, Research \& Evaluation. 2005; 10(7): 1-9.

21. Brown TA. Confirmatory factor analysis for applied research. Second ed. Boston, MA, USA: Guilford Publications; 2015.

22. Li CH. Confirmatory factor analysis with ordinal data: Comparing robust maximum likelihood and diagonally weighted least squares. Behav Res Methods. 2016; 48(3): 936-949.

23. Hu LT, Bentler PM. Cutoff criteria for fit indexes in covariance structure analysis: Conventional criteria versus new alternatives. Struct Equat Model: multi j. 1999; 6(1): 1-55.

24. Castilla H, Caycho T, Shimabukuro M, Valdivia A. Percepción del funcionamiento familiar: análisis psicométrico de la escala APGARfamiliar en adolescentes de Lima. Propósitos y Representaciones. 2014; 2(1):49-78.

25. Prazeres F, Santiago L. Relationship between health-related quality of life, perceived family support and unmet health needs in adult patients with multimorbidity attending primary care in Portugal: a multicentre cross-sectional study. Health Qual Life Outcomes. 2016; 14(1):156.

26. Pless IB, Satterwhite B. A measure of family functioning and its application. Soc Sci Med. 1973; 7(8):613-20.
27. Edman SO, Cole DA, Howard GS. Convergent and discriminant validity of FACES-III: family adaptability and cohesion. Fam Process. 1990; 29(1): 95-103.

28. Epstein N, Baldwin L, Bishop D. The McMaster family assessment device. J Marital Fam Therapy. 1983; 9(2): 171-180.

29. Reyes Romagosa DE, Torres Pérez I, Quesada Oliva LM, Milá Fernández M, Labrada Estrada HE. Hábitos bucales deformantes en niños de 5 a 11 años. Medisan. 2014; 18(5): 606-612.

30. Reisine ST, Psoter W. Socioeconomic status and selected behavioral determinants as risk factors for dental caries. J Dent Educ. 2001; 65(10): 1009-1016.

31. Han SH, Kim B, Lee SA, Korean Qo LiESG. Contribution of the family environment to depression in Korean adults with epilepsy. Seizure. 2015; 25: 26-31.

32. Lee MK, Yun YH. Family functioning predicts end-of-life care quality in patients with cancer: multicenter prospective cohort study. Cancer Nurs. 2017. DOI: 10.1097/NCC.0000000000000495.

33. Herrera Santí PM. La familia funcional y disfuncional, un indicador de salud. Rev Cubana Med Gen Integr. 1997; 13(6): 591-595. 